\title{
Local mean based adaptive thresholding to classify the cartilage and background superpixels
}

\author{
Hong-Seng Gan ${ }^{1}$, Bakhtiar Al-Jefri Adb Salam ${ }^{2}$, Aida Syafiqah Ahmad Khaizi ${ }^{3}$, \\ Muhammad Hanif Ramlee ${ }^{4}$, Wan Mahani Wan Mahmud ${ }^{5}$, Yeng-Seng Lee ${ }^{6}$, \\ Khairil Amir Sayuti ${ }^{7}$, Ahmad Tarmizi Musa ${ }^{8}$ \\ 1,2,3 Medical Engineering Technology Section, British Malaysian Institute, Universiti Kuala Lumpur, Malaysia \\ ${ }^{4}$ Medical Devices and Technology Group (MEDITEG), Sport Innovation and Technology Center (SITC), \\ Universiti Teknologi Malaysia, Malaysia \\ ${ }^{5}$ Biomedical Modelling and Stimulation (BIOMEMS) Research Group, Department of Electronic Engineering, \\ Faculty of Electrical and Electronic Engineering, Universiti Tun Hussein Onn Malaysia, Malaysia \\ ${ }^{6}$ Department of Electronic Engineering Technology, Faculty of Engineering Technology, \\ Universiti Malaysia Perlis, Malaysia \\ ${ }^{7,8}$ Department of Radiology, School of Medical Science, Universiti Sains Malaysia, Malaysia \\ ${ }^{8}$ Department of Radiology, Hospital Kuala Lumpur, Malaysia
}

\section{Article Info}

Article history:

Received Dec 16, 2018

Revised Feb 14, 2019

Accepted Feb 27, 2019

\section{Keywords:}

Adaptive threshold

Knee cartilage segmentation

Random walks

Seeds

\begin{abstract}
Semi-automatic segmentation is common in medical image processing because anatomical geometries demonstrated by human anatomical parts often requires manual supervision to provide desirable results. However, semi-automatic segmentation has been infamous for requiring excessive human intervention and time consuming. In order to reduce aforementioned problems, seed labels have been generated automatically using superpixels in our previous works. A fixed threshold method has been implemented to classify cartilage and background superpixels but this method is reported to lack the adaptiveness to changing image properties in 3D magnetic resonance image of knee. As a result, the coverage of background seeds are not sufficient to cover whole background area in some cases. In this work, we proposed a local mean based adaptive threshold method as a better alternative to the fixed threshold method. We calculated local mean for each block in an integral image and then use it to differentiate background superpixels from cartilage superpixels. The method is robust to illumination changes and simple to use. We tested the adaptive threshold on 35 knee images of different anatomical geometries and proved the proposed method could provide more comprehensive background seed labels distribution compared to fixed threshold method.
\end{abstract}

Copyright () 2019 Institute of Advanced Engineering and Science. All rights reserved.

\section{Corresponding Author:}

Hong-Seng Gan,

Medical Engineering Technology Section,

British Malaysian Institute, Universiti Kuala Lumpur,

53100 Gombak, Selangor, Malaysia.

Email: hongseng@unikl.edu.my

\section{INTRODUCTION}

Thresholding is a common image processing technique used to classify unlabeled pixels into different classes [1]. In general, there are two types of threshold approach in image processing i.e. global threshold and local threshold. Global threshold [2], also known as fixed threshold, is the most simple and straightforward threshold technique used for binary image segmentation by applying a general threshold value across the image. An effective fixed threshold method will require discriminative foreground and 
background image properties. Meanwhile, local threshold, or adaptive threshold, will implement several threshold values to different regions/ classes of the image. The threshold values will be determined by the region information of the neighboring pixels where the thresholding is applied.

One popular local threshold is Wellner's Method [3]. The local threshold method is straightforward and robust to variation in illumination, which is an important characteristic of medical images. An average of a predefined window is computed and defined around each pixel. Neighboring pixels of all sides are being considered to obtain a better average of image pixels. Then, integral image is applied to average the computation of image pixel in linear time. Compared to fixed threshold, adaptive threshold is more adaptive application-wise due to its flexible threshold setting. Therefore, the application of threshold method is wide; ranging from medical image segmentation [4], computer-aided diagnosis [5] to image denoising [6].

The segmentation of knee cartilage is challenging [7]. While automatic segmentation is desirable, it is not feasible due to the irregular anatomical geometry and thin structure of knee cartilage. Huge amount of training data will be the prerequisite to produce successful automatic segmentation models, in addition to heavy computational burden. As a result, semi-automatic knee cartilage segmentation methods have been viewed as the better alternative to automatic segmentation attributed to its direct expert intervention advantage to attain desirable results. There are several semi-automatic segmentation methods such as graph cuts [8], livewire [9] and random walks [10] which are applied in knee cartilage segmentation.

Nevertheless, most semi-automatic segmentation methods have reported major technical limitations. Graph cuts method has been sensitive to image noise and is usually limited to binary segmentation, which is not suitable for multilabel knee cartilage segmentation [11]. Livewire method is originated from Shortest Path algorithm but the algorithm is plagued by the "shortcut" problem. In order to overcome this limitation, excessive boundary points are required to guide the segmentation. This will be extremely tedious especially when handling the curvature region of cartilage. Finally, random walks method is robust to image noise and shortcut problem, in addition simultaneous multilabel segmentation. Unfortunately, the method is infamous to be sensitive to location of seed labels.

In our previous works, an automatic seed label generation model has been developed to overcome the problem faced by random walks segmentation. Fixed threshold methods has been utilized in the model to differentiate background from cartilage seed labels. Yet, fixed threshold method is not robust to the varying image superpixels' intensity properties. Hence, the objective of this study is to improve the automatic seed labels generation model's thresholding mechanism with Wellner based adaptive threshold.

\section{MATERIALS AND METHOD}

Knee image structure is complex since it consists of background and foreground objects which is tibial, patella and femoral cartilage as a whole. Non-cartilage background usually occupies most of the image and the knee cartilage is always positioned at the center of image [12]. Development of automatic seeds generation aims to substitute the background scribbling required to initialize conventional random walks segmentation [10-14]. For instance, random walks are extremely sensitive to placement of seed locations due to a lack of global feature distribution. Hence, we proposed to substitute manual background labelling with an automatic seed labels generation [15]. Figure 1 explains the advantage of the automatic system to overcome excessive user intervention.

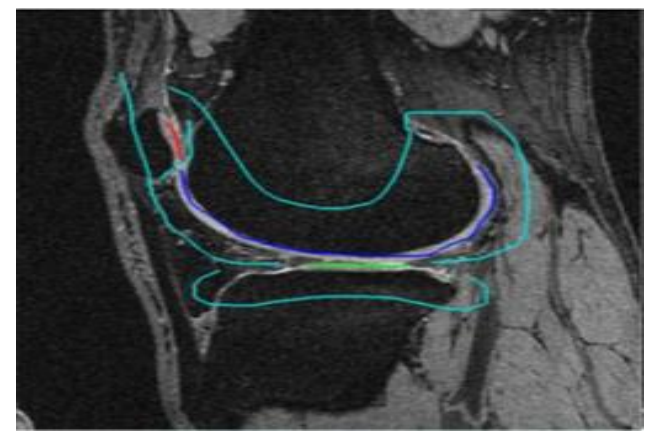

(a)

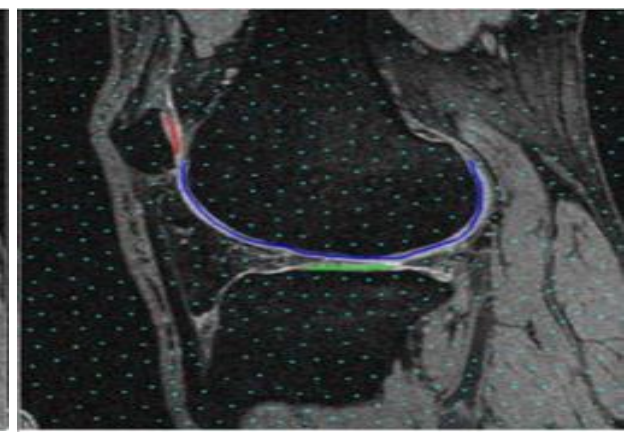

(b)

Figure 1. (a) Scribblings are required to initialize the random walks segmentation, but it is time consuming and remains sensitive to seed locations. (b) Use of background seeds saves scribbling time and reduce user interventions

Indonesian J Elec Eng \& Comp Sci, Vol. 15, No. 1, July 2019 : 221 - 230 


\subsection{Limitation in Automatic Seeds Generation}

While the automatic model [16] helps to reduce the excessive manual intervention problem, it is not without technical limitation. Currently, the model's fixed thresholding is derived from averaged histogram of superpixels' saliency values. The fixed threshold value was set at $\mathrm{T}=100$. But saliency detection often detect the object (cartilage) at the center of image; rendering the superpixels near to center location to possess higher saliency values. As a result, fixed threshold method is not robust enough in some occasional cases and create an empty "hole" phenomena in the center of image. Figure 2 demonstrates abovementioned phenomena in which the model fails to recognize background superpixels near to the center of image.

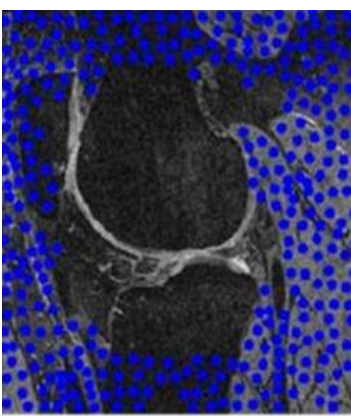

(a)

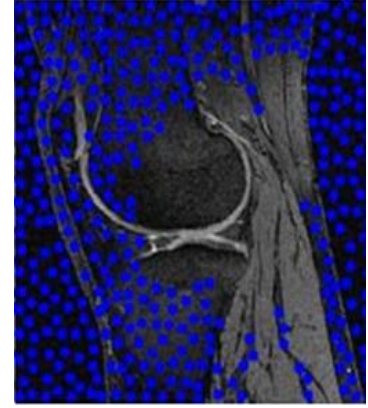

(b)

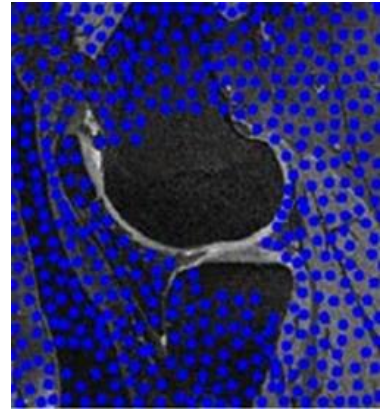

(c)

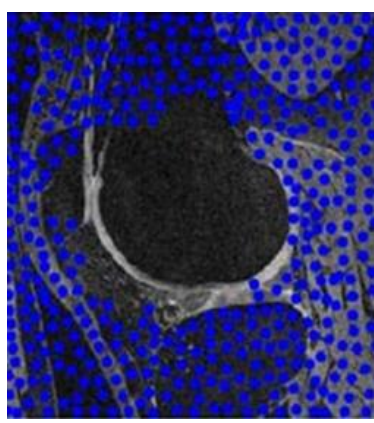

(d)

Figure 2. (a) - (d) Occasional failure cases encountered when using the automatic seeds generation model

\subsection{Local Mean-Based Adaptivr Threshold}

In this works, we replaced the fixed threshold with a local mean-based adaptive threshold using integral image. Instead of pixelwise intensity value, we used saliency value for each superpixel as threshold parameter [17]. Figure 3 demonstrates the feature map of superpixel-based MR image of knee using saliency values. An integral image, I is also known as a summed-area table where the cumulative sum of real numbers is computed over a rectangular region of the image.

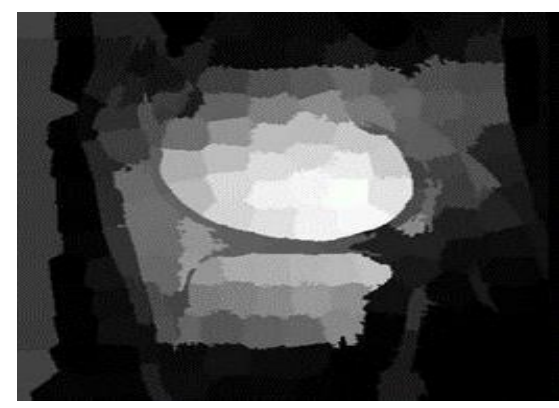

(a)

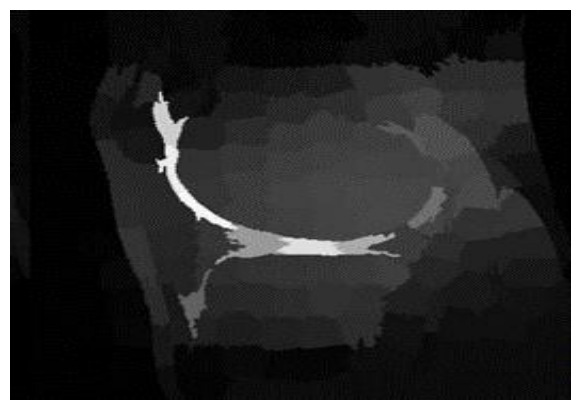

(b)

Figure 3. (a) First round of saliency detection using cartilage prior. (b) Second round of saliency detection using background priors

The saliency value, denoted as $f(x, y)$ at any location within the integral image, $I(x, y)$, is equivalent to the sum of all superpixels' values above and to the left of the superpixel, including the corresponding superpixel. The cumulative summation of all $(x$,$) terms at each location (x$,$) is given in (1)$.

$$
I(x, y)=f(x, y)+I(x-1, y)+I(x, y-1)-I(x-1, y-1)
$$

The main idea of this adaptive threshold is to compare each superpixel to an average of the surrounding superpixels. Let's set $\mathrm{P}(\mathrm{x}, \mathrm{y})$ as the original superpixel saliency value at superpixel $(\mathrm{x}, \mathrm{y})$ within an image of size $m \times n$. Then, a window with pre-defined two dimensional rectangular area $w$ is implemented. In this work, we defined the size of window, $w=15 \times 15$ and computed the mean value of each saliency value 
based on the integral image. The saliency values were averaged by the window. We set the superpixel threshold, $\mathrm{T}(\mathrm{x}, \mathrm{y})$ on whether it was lower than the $\mathrm{t}$ percent of average value of pixels within $\mathrm{w}$. In case of saliency value lower than the threshold, it was classified as 1 (black/ background) and vice versa. The threshold rule is given in (2).

$$
T(x, y)=\left\{\begin{array}{ccc}
1 & \text { if } & P(x, y)<\left(\frac{f(x, y)}{w}\right)\left(\frac{100-t}{100}\right) \\
0 & ; & \text { otherwise }
\end{array}\right.
$$

Figure 4 shows the use of integral image to compute the sum of pixels' values and the result of adaptive threshold.

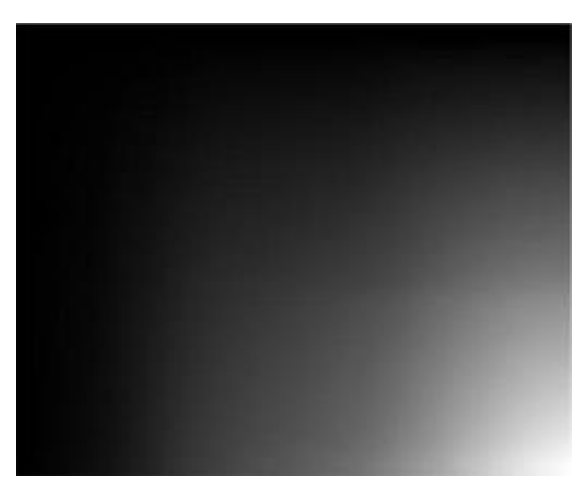

(a)

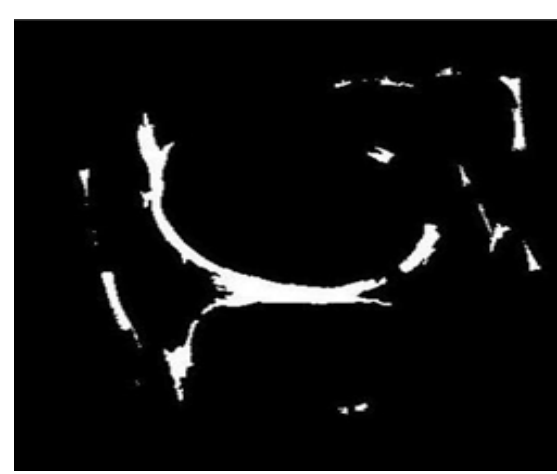

(b)

Figure 4. (a) Example of integral image produced from MR image of knee. (b) Example of binary image classification as a result of adaptive threshold

\subsection{Automatic Generation of Seeds}

After the classification of superpixels based on adaptive threshold, we ought to generate seeds on each background superpixel. To do so, Fuzzy C-Means (FCM) [18] was implemented to generate seeds. The objective function of FCM, $J_{m}$ was governed by a membership $\mu_{i j}^{m}$ in (3).

$$
J m=\sum_{i=1}^{N} \sum_{j=1}^{C} \mu_{i j}^{m}\|x i-C j\|^{2}
$$

Where the membership $\mu_{i j}^{m}$ evaluates the percentage of belonging to pixel $x_{i}$ to cluster $C_{j}$ using Euclidean distance, $d_{i j}=\sqrt{\sum x_{i}-C_{j}^{2}}$ and $m$ is the degree of membership.

Even though the cluster centroid did not directly represent any seed, we could approximate the nearest pixel to the centroid as seed using Euclidean distance as shown in (4).

$$
\operatorname{dist}(i j)=\min \sqrt{\sum x_{i}-C_{j}^{2}}
$$

A demonstration of seeds generation using the adaptive threshold classification is shown in Figure 5. We still reserved cartilage label for manual scribbling as human knowledge priori is critical to produce desirable results. The implementation of this work was conducted by using an inhouse developed medical image processing software [17-19].

\section{RESULTS}

\subsection{Image Dataset}

The knee images are taken from the Osteoarhtritis Initiative (OAI) dataset. Consents were obtained from all patients and approved by the Institutional Ethical Committee. A total of 35 knee images were used in this study. All images were acquired using 3.0T MRI scanner (Siemens Magnetom Trio, Erlangen, Germany) with quadrate transmit-receive knee coil (USA Instruments, Aurora, OH) [22]. The DESS images have 
section thickness of $0.7 \mathrm{~mm}$ and in-plane resolution $0.365 \times 0.456 \mathrm{~mm}^{2}$ (field of view $=140 \times 140 \mathrm{~mm}$, flip angle $=25^{\circ}, \mathrm{TR} / \mathrm{TE}=16.3 / 4.7 \mathrm{msec}$, matrix size $=384 \times 384 \mathrm{~mm}$, bandwidth $=185 \mathrm{~Hz} /$ pixel) [23]. The segmentation model was developed by using MATLAB R2014a (Mathworks, Natick, MA) on a laptop equipped with Core i7-4700HQ@2.50 GHz processor and 8.00 GB RAM.

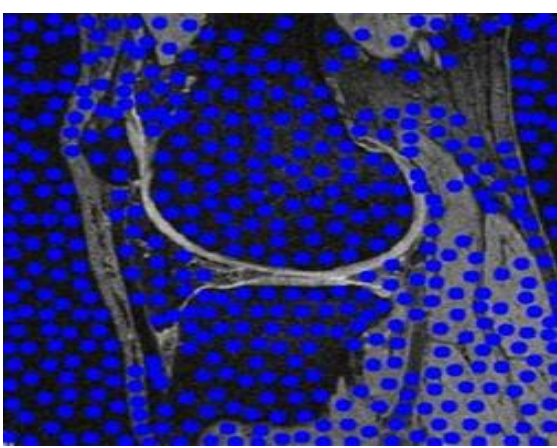

(a)

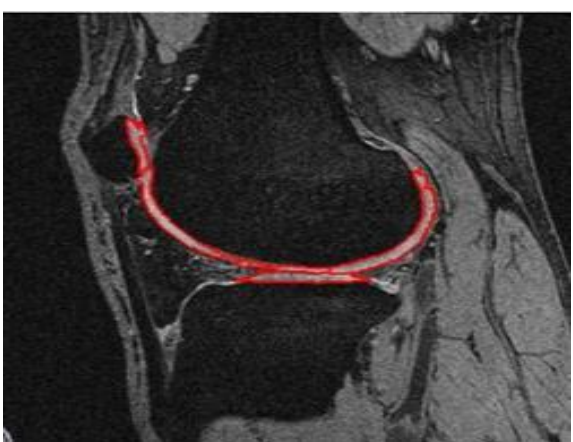

(b)

Figure 5. (a) Example of seeded image using adaptive threshold method, (b) Segmentation result of femoral, tibial and patellar cartilage

\subsection{Qualitative Results}

The effect of the proposed method is evaluated by comparing the distribution of background seed labels generated by using adaptive threshold method against fixed threshold method across the knee image. The aim of the qualitative evaluation is to examine the capability of the proposed adaptive threshold method to overcome the empty "hole" phenomena and fully enclose the background region with computer generated background seed labels; thus minimizing the need of manual intervention. The results of 10 knee images indicating before and after the implementation of the proposed method are demonstrated in Figure 6 . Since the anatomical geometry of knee cartilage varies across image slices, we have included the implementation of adaptive threshold method across different knee image slices.

\section{DISCUSSION}

Based on the results, we can observe obvious improvement in background seeds label distribution across the knee image when adaptive threshold method was applied to the automatic seed labels generation model. The reliability of the implementation is confirmed by applying the method across different knee cartilage geometries. Comparatively, automatic seed generation model using fixed threshold method often fails to create background labels at the center location of the knee image. This failure is mainly due to the higher saliency values demonstrated by background superpixels located near to the center position. Therefore, fixed threshold method fails to recognize those background superpixles with higher saliency values.

On the other hand, automatic seeds generation model using adaptive threshold method can easily resolve the phenomena reported by the fixed threshold method. As shown in Figure 6, background superpixels located at the center of image have been covered with background seeds successfully. For instance, adaptive threshold method has been found to be more robust to varying image property such as shape, intensity, the presence of noise and uneven background. The fixed threshold is more effective when the intensity distribution between knee cartilage and background are clearly distinct and thus, the selection of a general threshold value can fulfill the overall image property condition. In this context, we can observe the use of Wellner based adaptive threshold proves to be more adaptive to varying saliency values possessed by the background superpixels located near to the center of image, where the cartilage superpixels are located.

Although our study has suggested that the adaptive threshold method can provide better seeds label distribution across the image background, there are several limitations in this study. First, there is no categorization of knee image into healthy and abnormal knee cartilage. This is attributed to the focus of this paper is to assess the distribution of background seeds instead of cartilage. Hence, the influence of pathological features such as osteophytes, bone marrow lesions and effusion are minimal. Second, we did not conduct quantitative analysis in this paper. Nonetheless, the absence of quantitative analysis in this study still follows strictly the standard evaluation protocol of threshold-based analysis from existing studies [24-25]. 
Besides, the aim of this study is determine the capability of the adaptive threshold to provide comprehensive seeds label distribution. Thus, it is difficult to apply evaluation parameters such as Dice Similarity Coefficient (DSC) which requires at least two object classes. Nonetheless, qualitative analysis by comparing the implementation of adaptive threshold and fixed threshold method has demonstrated the superior performance reported by the adaptive threshold method.

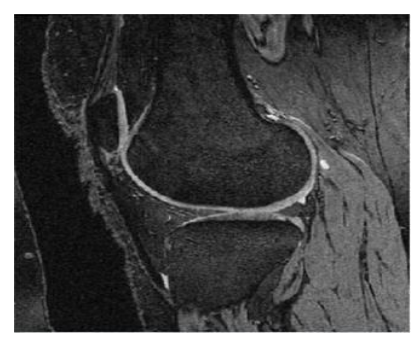

(a)

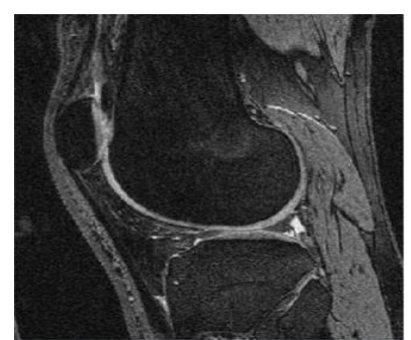

(d)

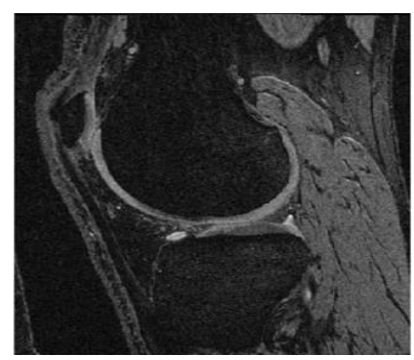

(g)

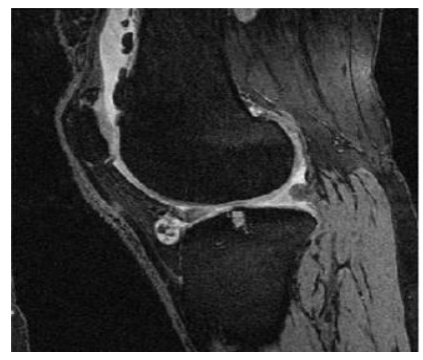

(j)

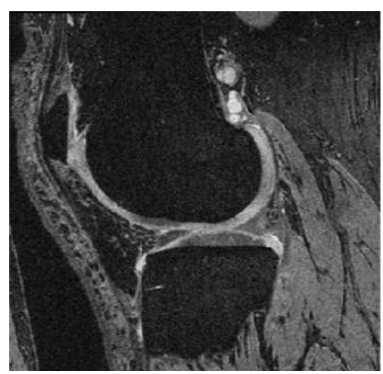

(m)

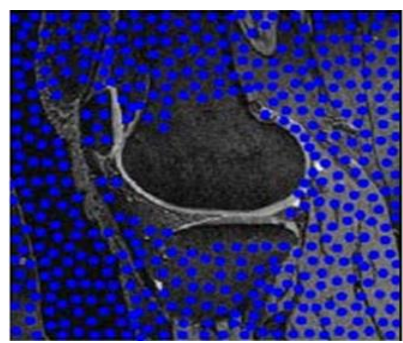

(b)

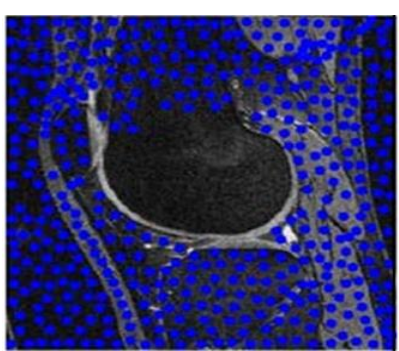

(e)

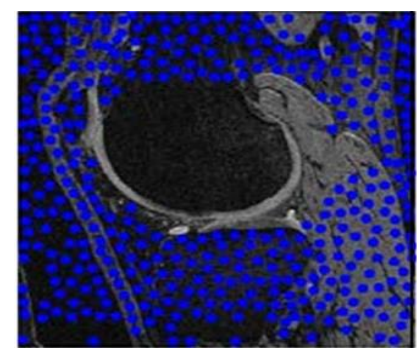

(h)

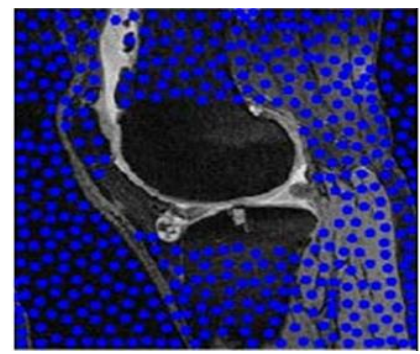

(k)

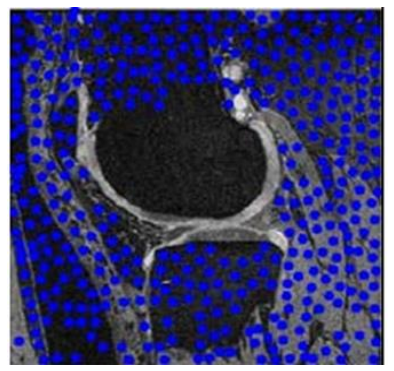

(n)

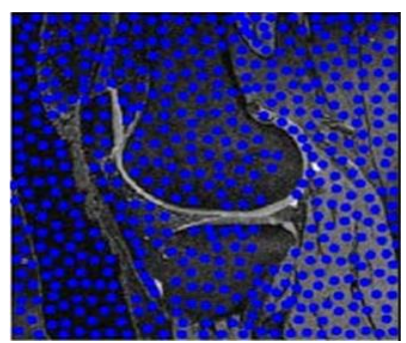

(c)

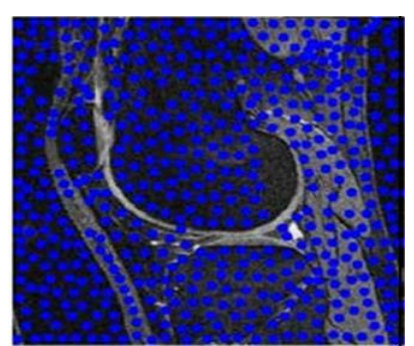

(f)

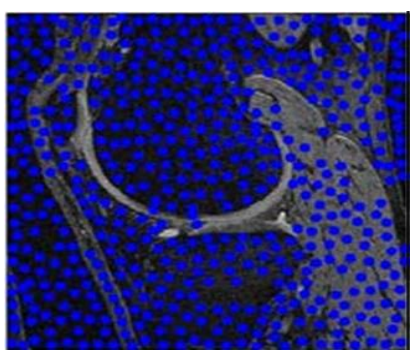

(i)

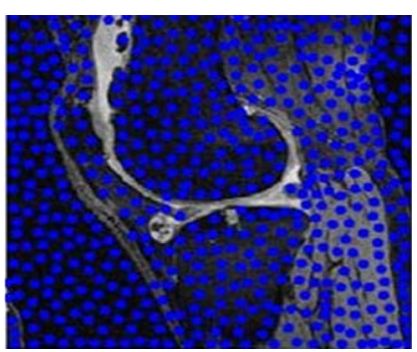

(1)

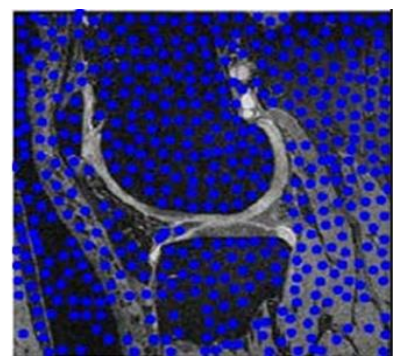

(o) 


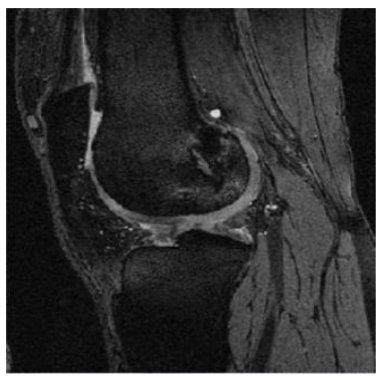

(p)

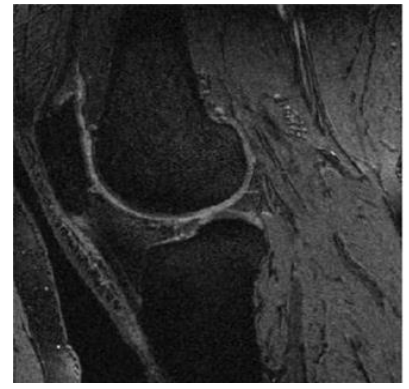

(s)

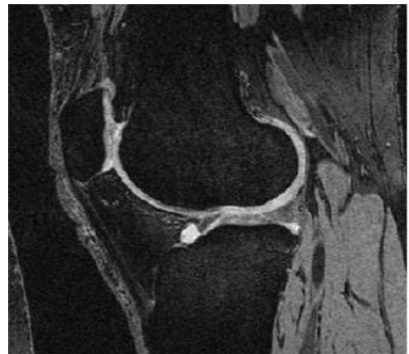

(v)

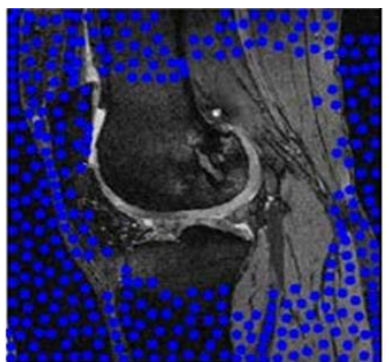

(q)

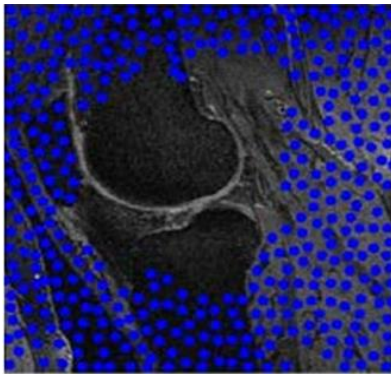

(t)

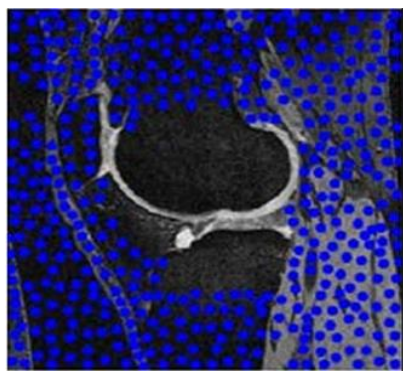

(w)

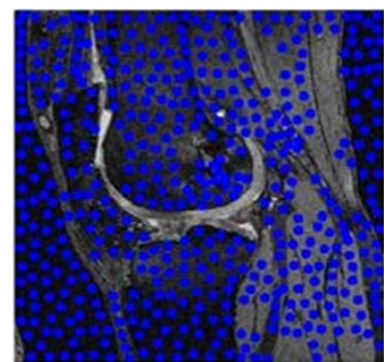

(r)

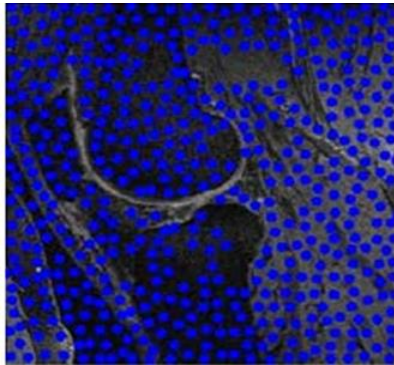

(u)

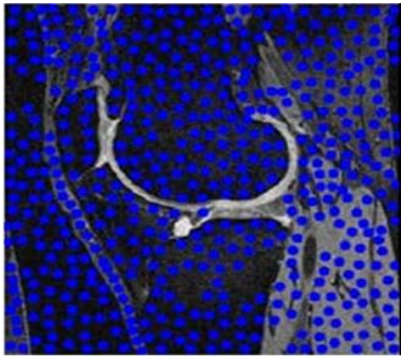

(x)

Figure 6. Effect of adaptive threshold on the automatic seeds generation model. (a), (d), (g), (j), (m), (p), (s) and (v): Original MR image of knee. (b), (e), (h), (k), (n), (q), (t) and (w): Seeds distribution using fixed threshold method. (c), (f), (i), (l), (o), (r), (u) and (x): Seeds distribution using the adaptive threshold method.

\section{CONCLUSION}

The study concluded the successful use of adaptive threshold as a better alternative to fixed threshold method as implemented previously in out automatic seeds generation model. The advantage illustrated by the adaptive threshold method minimizes the need for additional manual intervention. Future works should focus on conducting further quantitative analysis to strengthen the advantage of adaptive threshold and also expanding the automation of seed generation to different types of knee cartilage i.e. femoral, tibial and patellar cartilage.

\section{ACKNOWLEDGEMENT}

The authors gratefully acknowledge the Fundamental Research Grant Scheme (FRGS) Grant Number: FRGS/1/2018/ICT02/UNIKL/02/4 (Project title: Fundamental Study on Graph Transformed Deep 'Interactive' Learning Framework in Medical Image Segmentation) provided by the Malaysian Ministry of Education (MoE) and the University Short Term Research Grant (STRG) (Grant number: str17015) provided by Centre of Research and Innovation (CoRI), and Universiti Kuala Lumpur-British Malaysian Institute (UniKL BMI) in supporting this study. 


\section{REFERENCES}

[1] M. Luessi, M. Eichmann, G. M. Schuster, and A. K. Katsaggelos, "Framework for efficient optimal multilevel image thresholding," 2009, pp. 013004-1-013004-10.

[2] M. Sezgin and B. Sankur, "Survey over image thresholding techniques and quantitative performance evaluation," 2004, pp. 1-20.

[3] D. Bradley and G. Roth, "Adaptive Thresholding using the Integral Image," Journal of Graphics Tools, vol. 12, pp. 13-21, 2007.

[4] C. Kim, J. Yoon, and Y. Lee, "Medical Image Segmentation by More Sensitive Adaptive Thresholding," in 2016 6th International Conference on IT Convergence and Security (ICITCS), 2016, pp. 1-3.

[5] A. Issac, M. Partha Sarathi, and M. K. Dutta, "An adaptive threshold based image processing technique for improved glaucoma detection and classification," Computer Methods and Programs in Biomedicine, vol. 122, pp. 229-244, 2015/11/01/ 2015.

[6] L. Liu, Z. Jia, J. Yang, and N. Kasabov, "A medical image enhancement method using adaptive thresholding in NSCT domain combined unsharp masking," International Journal of Imaging Systems and Technology, vol. 25, pp. 199-205, 2015.

[7] J. Kubicek, M. Penhaker, M. Augustynek, I. Bryjova, and M. Cerny, "Segmentation of Knee Cartilage: A Comprehensive Review," Journal of Medical Imaging and Health Informatics, vol. 8, pp. 401-418, // 2018.

[8] Y. Y. Boykov and M. P. Jolly, "Interactive graph cuts for optimal boundary and region segmentation of objects in N-D images," in IEEE International Conference on Computer Vision, Vancouver, Canada, 2001, pp. 105-112.

[9] A. X. Falcão and J. K. Udupa, "A 3D generalization of user-steered live-wire segmentation," Medical Image Analysis, vol. 4, pp. 389-402, 12// 2000.

[10] L. Grady, "Random walks for image segmentation," IEEE Transactions on Pattern Analysis and Machine Intelligence, vol. 28, pp. 1768-1783, 2006.

[11] Y. Boykov and M.-P. Jolly, "Interactive Organ Segmentation Using Graph Cuts," in Medical Image Computing and Computer-Assisted Intervention - MICCAI 2000. vol. 1935, S. Delp, A. DiGoia, and B. Jaramaz, Eds., ed: Springer Berlin Heidelberg, 2000, pp. 276-286

[12] H. S. Gan, R. A. M. Rosidi, H Hamidun \& K.A. Sayuti, M.H. Ramlee \& A.H.A. Karim \& B.A. A Salam. Binary Seeds Auto Generation Model for Knee Cartilage Segmentation. 1-5. 10.1109/ICIAS.2018.8540570. 2018.

[13] H. S. Gan, T. S. Tan, K. A. Sayuti, A. H. A. Karim, and M. R. A. Kadir, "Multilabel graph based approach for knee cartilage segmentation: Data from the osteoarthritis initiative," in Biomedical Engineering and Sciences (IECBES), 2014 IEEE Conference on, 2014, pp. 210-213.

[14] G. Hong Seng, S. Khairil Amir, and A. K. Ahmad Helmy, "Investigation of Random Walks Knee Cartilage Segmentation Model using Inter-Observer Reproducibility: Data From The Osteoarthritis Initiative," Bio-Medical Materials and Engineering, vol. 28, pp. 75-85, 2017.

[15] H. S. Gan, T. S. Tan, A. H. A. Karim, K. A. Sayuti, and M. R. A. Kadir, "Interactive medical image segmentation with seed precomputation system: Data from the Osteoarthritis Initiative," in Biomedical Engineering and Sciences (IECBES), 2014 IEEE Conference on, 2014, pp. 315-318.

[16] G. Hong Seng and S. Khairil Amir, "Comparison of Improved Semi-Automated Segmentation Technique with Manual Segmentation: Data from the Osteoarthritis Initiative," American Journal of Applied Sciences, vol. 13, pp. 1068-1075, 2016.

[17] R. Achanta, "SLIC superpixels compared to state-of-the-art superpixel methods," IEEE Transactions on Pattern Analysis and Machine Intelligence, vol. 34, pp. 2274-2282, 2012.

[18] J. Bezdek, R. Ehrlich, and W. Full, "FCM: the fuzzy c-means clustering algorithm," Computers \& Geosciences, vol. 10, pp. 191-203, 1984.

[19] G. Hong-Seng, T. Tian-Swee, W. Liang-Xuan, T. Weng-Kit, K. A. Sayuti, A. H. A. Karim, et al., "Interactive knee cartilage extraction using efficient segmentation software: Data from the osteoarthritis initiative," Bio-Medical Materials and Engineering, vol. 24, pp. 3145-3157, 2014.

[20] H.-S. Gan, A. H. A. Karim, K. A. Sayuti, T.-S. Tan, and M. R. A. Kadir, "Analysis of parameters' effects in semiautomated knee cartilage segmentation model: Data from the osteoarthritis initiative," in AIP Conference Proceedings, Songkhla, Thailand, 2016, pp. 030052-1 - 030052-6.

[21] A. S. A. Khaizi, R. A. M. Rosidi, H. S. Gan, and K. A. Sayuti, "A mini review on the design of interactive tool for medical image segmentation," in 2017 International Conference on Engineering Technology and Technopreneurship (ICE2T), 2017, pp. 1-5.

[22] F. Eckstein, M. Hudelmaier, W. Wirth, B. Kiefer, R. Jackson, J. Yu, et al., "Double echo steady state magnetic resonance imaging of knee articular cartilage at 3 Tesla: a pilot study for the Osteoarthritis Initiative," Annals of the Rheumatic Diseases, vol. 65, pp. 433-441, 2006.

[23] C. G. Peterfy, E. Schneider, and M. Nevitt, "The osteoarthritis initiative: report on the design rationale for the magnetic resonance imaging protocol for the knee," Osteoarthritis and Cartilage, vol. 16, pp. 1433-1441, 2008.

[24] D. Zhang, X. Ye, and X. Hu, "An Improved Algorithm Based on Wellner's Threshold Segmentation Method," The Open Cybernetics \& Systemics Journal, vol. 9, pp. 32-36, 2015.

[25] L. Denghui and W. Yanhong, "Application of an improved threshold segmentation method in SEM material analysis," IOP Conference Series: Materials Science and Engineering, vol. 322, p. 022057, 2018. 


\section{BIOGRAPHIES OF AUTHORS}
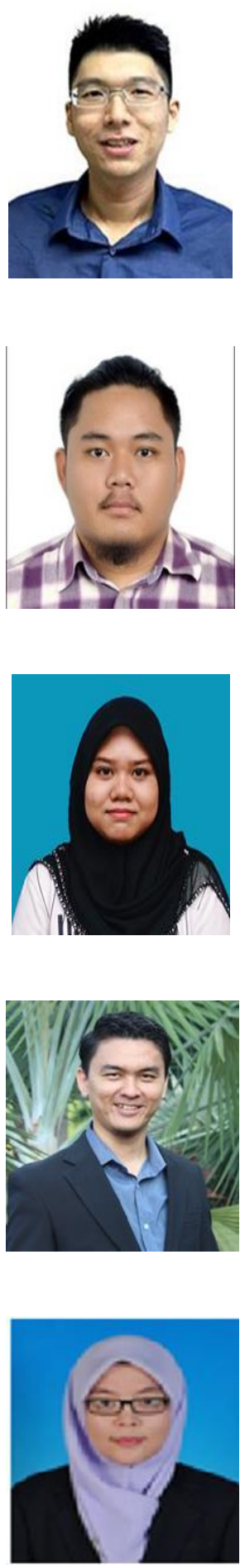

Wan Mahani Hafizah Wan Mahmud received her PhD in Biomedical Engineering from the Universiti Teknologi Malaysia in 2013. She started working as a Lecturer in Universiti Tun Hussein Onn Malaysia in 2014. Her research interests includes ultrasound imaging, medical image analysis, as well as e-health and rehabilitation system. She is a member of the Board of Engineers Malaysia (BEM).

Hong-Seng Gan received his $\mathrm{PhD}$ in Biomedical Engineering from the Universiti Teknologi Malaysia in 2016. Upon graduation, he joined the Universiti Kuala Lumpur as an Assistant Professor. His research interests covers medical image analysis and biomedical signal processing. He is a member of IEEE Engineering in Medical and Biology Society (EMBS), the Osteoarthritis Research Society International (OARSI) and the Board of Engineers Malaysia (BEM).

Bakthiar Al-Jefri Abd Salam receive his Bachelor of Engineering Technology in Medical Electronics from Universiti Kuala Lumpur British Malaysian Institute in 2018. He currently working as a biomedical engineer with Advance Pact Sdn Bhd at Hosital Permai Johor Bahru. $\mathrm{He}$ is a member of the Board of Engineers Malaysia (BEM).

Aida Syafiqah Ahmad Khaizi receive her Bachelor of Engineering Technology in Medical Electronics from Universiti Kuala Lumpur British Malaysian Institute in 2017. She currently a master of Engineering Technology in Electrical and Electronics student from univerisiti Kuala Lumpur British Malaysian Institute. Her research interest cover medical image processing. She is a member of the Board of Engineers Malaysia (BEM).

Muhammad Hanif Ramlee received his $\mathrm{PhD}$ in Biomedical Engineering from the Universiti Teknologi Malaysia (UTM) in 2015 after completing one-year research attachment at the Technical University of Madrid, Spain. Upon graduation, he joined the Universiti Kebangsaan Malaysia (UKM) as a post-doctoral researcher and then returned to UTM as a senior lecturer in 2016. His research interests cover biomechanics, biomaterials, rehabilitation engineering and biomedical design. He is a member of Board of Engineers Malaysia (BEM).

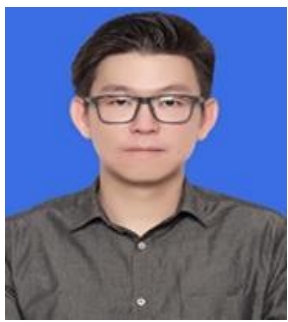

Yeng Seng received his Ph.D. in communication engineering from the School of Computer and Communication Engineering at Universiti Malaysia Perlis (UniMAP), Malaysia. He is a Senior Lecturer at the Department of Electronic Engineering Technology, Faculty of Engineering Technology, Universiti Malaysia Perlis (UniMAP), Malaysia. His research interests include Dielectric material characterization, Microwave absorber, Frequency Selective Surface (FSS), and Antenna. He also a member of IET, BEM, MBOT, IEEE and IEEE Microwave Theory and Techniques Society. 

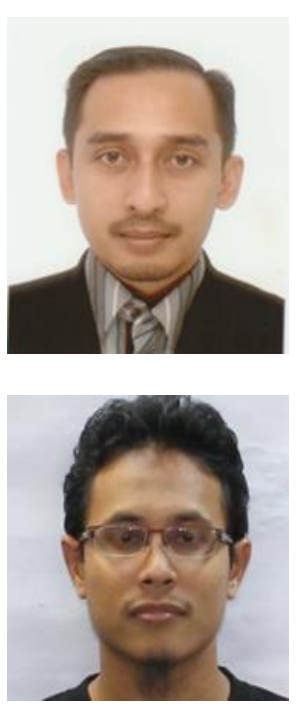

Khairil Amir received his Master of Medicine (Radiology) from the Universiti Sains Malaysia in 2012. After graduation, he joined the School of Medical Sciences, Universiti Sains Malaysia as the Lecturer and Clinical Radiologist. He subspecialised in Cardiovascular Imaging at the National Heart Institute, Kuala Lumpur in 2015 and the Royal Brompton Hsopital, Imperial College, London in 2016. He also collaborated in research on medical image analysis and biomedical signal processing with other universities. He is a registered member of Malaysian Medical Council (MMC), National Specialist Registry (NSR), National Conjoint Board for Radiology, Society for Cardiovascular Magnetic Resonance (SCMR) and an associate member of Malaysian Association of Medical Physics (MAMP).

Ahmad Tarmizi Musa received his Master of Medicine (Radiology) from Universiti Sains Malasyia. Currently working as medical lecturer at Universiti Sains Malasyia. He also collaborated in research on medical image analysis and biomedical signal processing with other universities. 